\title{
Research on the Remaining Load Forecasting of Micro-Gird based on Improved Online Sequential Extreme Learning Machine
}

\author{
Shaomin Zhang ${ }^{1, ~ a ~}$, Peng Zhou ${ }^{2, b}$, Baoyi Wang ${ }^{3, c}$ \\ 1, 2, ${ }^{3}$ School of Control and Computer Engineering, North China Electric Power University, Baoding, \\ 071003, China
}

aemail: zhangshaomin@126.com, bemail: zhoupengbest@qq.com, email: wangbaoyiqj@126.com

Keywords: ELM, Remaining Load Forecasting, Online Sequential, Micro-Grid

\begin{abstract}
In order to improve the forecast accuracy and stability of the micro-grid uncontrollable remaining load and provide a more reliable basis for micro-grid power generation plan, an ultra-short-term micro-grid uncontrollable remaining load forecasting model based on the improved online sequential Extreme Learning Machine is proposed. Aimed at the wind and solar power generation and load characteristics, the weight update of old and new training data is added to the Extreme Learning Machine. And the average value of multi-module is used to enhance the predict stability of the algorithm. After the real data from UCI Machine Learning Repository is analyzed, the result shows that the algorithm is superior to the traditional Extreme Learning Machine (ELM) and the online sequential Extreme Learning Machine (OS-ELM) and the proposed algorithm is feasible.
\end{abstract}

\section{Introduction}

The micro-grid has been a hot topic of power system [1]. Micro-grid can be seen as the "power" can also be seen as a "burden". So it could be run standalone in some special places like in remote mountain areas or islets [2]. Micro-power supply of micro-grid mainly includes two categories [3] which are controllable and uncontrollable micro-power supply. Controllable micro-power supply ensures stability and regulation, to achieve stable and efficient operation of independent micro-grid [4]. Uncontrollable micro-power supply mainly includes wind turbines and solar panels.

Due to the small scale of micro-grid, so accurately ultra-short-term remaining load forecast can be said to be the micro-grid system planning basis. Yan Gang put forward a kind of prediction method based on Improved LS-SVM algorithm [5]. Algorithm based on evolutionary neural network and the short-term power load forecasting model of chaotic feature selection is put forward by Sajjad Kouhi [6]. Although both SVM and evolutionary Neural Networks have strong nonlinear identification ability, good accuracy, and good global search ability. There are defects such as week generalization ability and slow training speed. Guang-Bin Huang put forward a new single hidden layer neural networks algorithm, called the Extreme Learning Machine (ELM) [7]. ELM and its extension have obtained good effect in wind [8] or solar [9] power prediction and load forecasting [10]. ELM has improved rapidly in training speed, but still need to be repeated scanning history data and update model to achieve the ideal predictive effect. The literature [11] put forward a kind of online sequential ELM (OS-ELM). When updating model, just enter the current training data blocks, without repeated scanning the historical data. Based on the literature [11], Sun Yigang came up with weighted OS-ELM (WOS-ELM) for discovering fault diagnosis of aircraft engine sensor [12].

Considering the old and new data and contingency of predicting remaining load, there came up with the concept of uncontrollable remaining load and multiple models WOS-ELM (MM-WOS-ELM) prediction algorithm, according to the literature [13] proposed the concept of remaining load. Adding the weight update of new and old training data on the basis of OS-ELM made the prediction model more focus on training data which had higher predicting accuracy. Because of predict accident of OS-ELM, there could be more than one model of OS-ELM and made the mean value of multiple models output as the final output of the prediction model. This paper 
analyzes power load of multiple families and real wind data and illumination intensity per minute in micro-grid and compares with the predictive result of ELM, OS-ELM and WOS-ELM.

\section{The Micro-Grid Uncontrollable Remaining Load}

The remaining load of micro-grid refers to the difference between the total of all loads with the sum of all output power of micro-powers.

Accurate ultra-short-term load forecasting uncontrollable micro-power supply can provide decision basis for generation of controllable micro-power. When the value is greater than 0 , show the generation of uncontrollable micro-power cannot be met by total electricity load, it requires other micro-power supply. But when the value is less than 0 , show the generation can.

The uncontrollable micro-power supply is very common by using wind or solar. The power output characteristics can be show through their mathematical model respectively. Their power model is important basis of forecasting. The formula of generated power $\boldsymbol{P}_{\text {wind }}$ which is changed with wind speed $v_{\text {wom }}$ is described as follows [14],

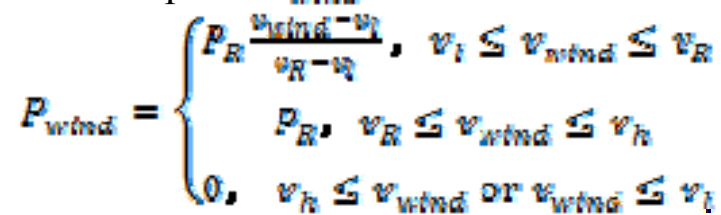

with $\boldsymbol{P}_{k}$ as normal power output of wind turbine, $v_{l}$ as cut-in wind speed, $v_{k}$ as cut-out wind speed and $v_{R}$ as rated wind speed.

Rated power output of photovoltaic power generation is calculated in the standard state. The output power formula of photovoltaic power generation is described as follows [15],

$$
P_{g}=\left\{\begin{array}{c}
P_{S T C} \frac{G_{s}}{G_{S T C}}\left(1+k\left(T_{C}-T_{R}\right)\right), G_{g}>C \\
0, G_{g} \leq C
\end{array}\right.
$$

with $P_{z}$ as the output power when light intensity is $G_{s}, P_{s r c}$ as the maximum output power under standard state, $G_{s}$ as real-time light intensity, $G_{\text {STTe }}$ as the light intensity under standard state, $k$ as the output correlation coefficient of temperature and power. $T_{C}$ as real-time temperature of photovoltaic cells, $T_{R}$ as the temperature under standard state and $C$ which is constant as the basic threshold of light intensity.

\section{The Online Sequential Extreme Learning Machine}

OS-ELM makes the training data block as the unit and adds new historical data block to training network which avoids consuming a lot of time in training. OS-ELM can be divided into two steps as a whole, including initial stage and continuous learning stage.

In initial stage, setting the number of hidden layer nodes $(L)$ and making a portion of the training sample as initialize learning. If the count of initial training sample is $N_{o}, N_{o} \geq L$ is necessary. Then, weight vector $\left(\boldsymbol{W}_{j}\right)$ and bias $\left(b_{j}\right)$ in hidden layer are generated, and calculating initial output matrix $\left(\boldsymbol{H}_{\mho}\right)$ in hidden layer. The initial output weight vector $\left(\boldsymbol{\beta}_{\cup}\right)$ could be calculated as follows,

$$
\boldsymbol{\beta}_{0}=\boldsymbol{P}_{0} H_{0}^{T} T_{0}=\left(H_{0}^{T} H_{0}\right)^{-1} H_{0}^{T} T_{0}
$$

with $\boldsymbol{T}_{0}$ as expectations.

In continuous learning stage, suppose the first $k+1$ training data is

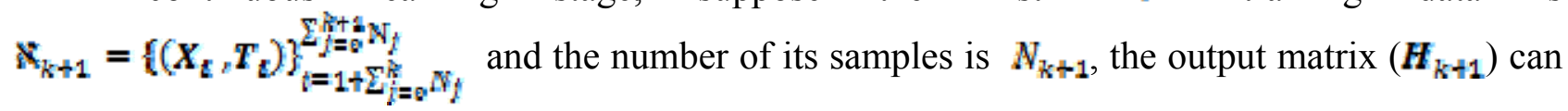
be confirmed. Now suppose the first $k$ training data has successfully enter the model, and there is $\boldsymbol{K}_{k}=\boldsymbol{H}_{k}^{\boldsymbol{T}} \boldsymbol{H}_{k}$. When the first $k+\mathbb{1}$ data block compares to input, $\boldsymbol{K}_{k+1}$ can be represented by $\boldsymbol{H}_{k}$ and $\boldsymbol{H}_{k+1}$ as follows,

$$
\boldsymbol{K}_{k+1}-\left[\begin{array}{c}
\boldsymbol{H}_{k} \\
\boldsymbol{H}_{k+1}
\end{array}\right]^{T}\left[\begin{array}{c}
\boldsymbol{H}_{k} \\
\boldsymbol{H}_{k+1}
\end{array}\right]-\boldsymbol{K}_{k}+\boldsymbol{H}_{k+1}^{T} \boldsymbol{H}_{k+1}
$$


Now, $\boldsymbol{K}_{k+1}^{-1}=\boldsymbol{P}_{k+1}$ and updating output bias as follows,

$\boldsymbol{\beta}_{k+1}=\boldsymbol{\beta}_{k}+\boldsymbol{P}_{k+1} \boldsymbol{H}_{k+1}^{\boldsymbol{T}}\left(\boldsymbol{T}_{k+1}-\boldsymbol{H}_{k+1} \boldsymbol{\beta}_{k}\right)$

Because calculating inverse matrix of $\boldsymbol{K}_{k+\text { : }}$ can cost a lot of time, in order to avoid inverting matrix and $\boldsymbol{K}_{k \mid 1}^{-1}=\boldsymbol{P}_{k \mid 1}$, according to Eq. (4), there is a formula as follows

$\boldsymbol{P}_{k+1}=\boldsymbol{P}_{k}-\boldsymbol{P}_{k} \times \boldsymbol{H}_{k+1}^{T} \times\left(\boldsymbol{I}+\boldsymbol{H}_{k+1} \times \boldsymbol{P}_{k} \times \boldsymbol{H}_{k+1}^{T}\right)^{-1} \times \boldsymbol{H}_{k+1} \times \boldsymbol{P}_{k}$

Then $k=k+1$ and continue, until the next training data block is none.

\section{Algorithm Design Based on the Multi-Module Weighted Online Sequential ELM}

Basis on Eq. (4), the matrix, which has old and new training data can be added weight on, is shown as follows,

$$
\boldsymbol{K}_{k+1}=\left[\begin{array}{c}
\boldsymbol{H}_{k} \\
\boldsymbol{\alpha} \boldsymbol{H}_{k+1}
\end{array}\right]^{z}\left[\begin{array}{c}
\boldsymbol{H}_{k} \\
\boldsymbol{\alpha} \boldsymbol{H}_{k+1}
\end{array}\right]
$$

where $\alpha$ is weight of new data block which is decided by last Root-Mean-Square Error (RMSE).

If RMSE $\in[a, b], \alpha=1$. If RMSE $\alpha a, \alpha=0.995$. If RMSE $>b, \alpha=1.005$. Calculating Eq. (7) and get function as follows,

$$
\boldsymbol{K}_{k+1}=\boldsymbol{K}_{k}+\sigma^{2} \boldsymbol{H}_{k+1}^{F} \boldsymbol{H}_{k+1}
$$

Inversing Eq. (8) on both sides of the equal sign and $K_{k+1}^{-1}=P_{k+1}$ and $K_{k}^{-1}=P_{k}$, there are equation as follows,

$$
\boldsymbol{P}_{k+1}=\boldsymbol{P}_{k}-\boldsymbol{P}_{k} \times \boldsymbol{H}_{k+1}^{T} \times\left(\boldsymbol{I} / \alpha+\boldsymbol{H}_{k+1} \times \boldsymbol{P}_{k} \times \boldsymbol{H}_{k+1}^{T}\right)^{-1} \times \boldsymbol{H}_{k+1} \times \boldsymbol{P}_{k}
$$

In this paper, the WOS-ELM model is processed aimed at the predict error.

Multiple model prediction is combined with WOS-ELM modules which number is M. To avoid the error of output is too large, figuring up the average of all WOS-ELM modules output as follows,

$$
o_{i}=\frac{1}{B S} \sum_{i=1}^{S v} o_{t}^{t}
$$

where $o_{i}^{j}$ is the first $\mathrm{j}$ model output.

The main idea of MM-WOS-ELM is to update weights and to calculate the average of modules.

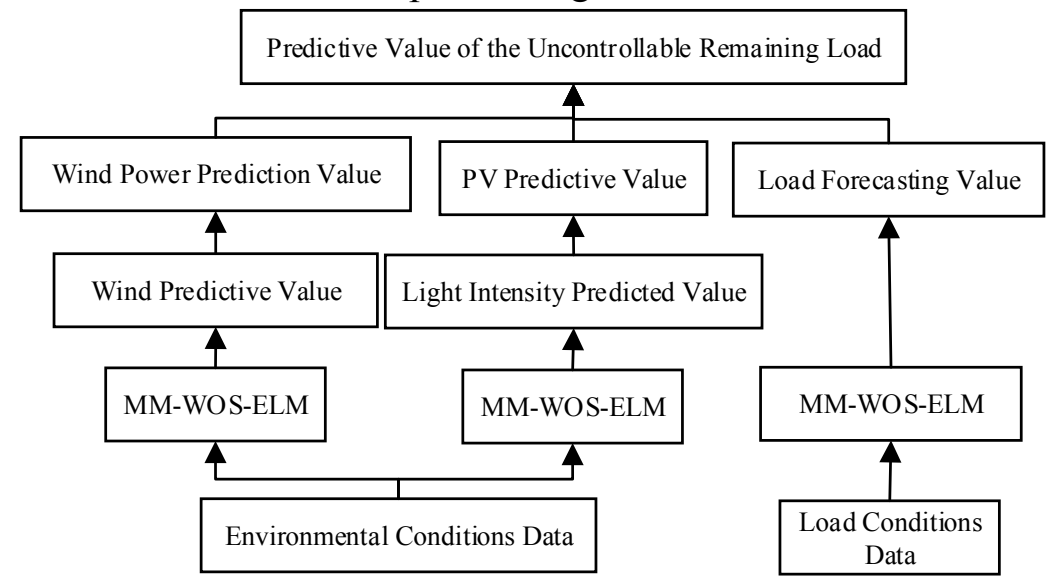

Fig.1. Uncontrollable remaining load forecasting model based on MM-WOS-ELM

The specific steps of MM-WOS-ELM are shown as follows,

(1) Getting historical training data sets and the data sets were input to WOS-ELM modules.

(2) According to the WOS-ELM training logic, train these WOS-ELM modules respectively.

(3) After getting results of modules, the average must be calculated. This value is regarded as the final prediction value of the whole model.

(4) Slide along the data time axis and access to new historical data, each WOS-ELM modules update their own training data right weight values based on the last real historical data. Then return to the step (1) for next circulation.

The uncontrollable remaining load forecasting model based on MM-WOS-ELM is shown in Figure 1. 


\section{Experiment and Example Analysis}

The remaining load forecasting accuracy uses the Root Mean Square Error (RMSE) for testing indicators as follows,

$$
\text { RMSE }=\sqrt{\frac{1}{H} \sum_{t=1}^{N}\left(\theta_{t}-T_{t}\right)^{2}}
$$

where $N$ is the number of prediction values one time, $o_{t}$ is the first t prediction value and $T_{t}$ is the first $\mathrm{t}$ true value.

The stability of the algorithm uses the Standard Deviation (SD) as a measure. The formula is described as follows,

$$
\mathrm{SD}=\sqrt{\frac{1}{i f} \sum_{t=1}^{N}\left(R M S E_{t}-\text { RMSE }^{2}\right.}
$$

where $N$ is the total number of test errors, $R M S E_{t}$ is the first $\mathrm{i}$ of RMSE and $R M S E$ is the average of RMSE under several tests.

Considering the algorithm running time, forecasting accuracy and forecast stability, add the three normalized data as a comprehensive index.

Syn $=T$ tme norm $_{\text {nom }}+R M S E_{\text {norm }}+S D_{\text {nerm }}$

with $\$ y n$ as comprehensive index of algorithm, Ttme $\boldsymbol{s}_{\text {nemm }}$ as algorithm running time, $R M S E_{\text {worm }}$ as the RMSE of prediction algorithm and $S D_{\text {nomm }}$ as the SD of forecasting accuracy.

The input samples include two classes. One is the training data set of load which includes date, time and temperature. Another is the training data set of environment including date, time, temperature, precipitation and atmospheric humidity. Both two training data sets are normalized. The experimental targets are predicting wind speed, light intensity and electricity load, and then calculate the uncontrollable remaining load.

Micro-grid data, which is from 11:45 on March 13, 2012 to 2:00 on April 4 provided by UCI Machine Learning Repository website, used in this experiment. Determining the number of WOS-ELM modules based on integrated indicators. And MM-WOS-ELM was compared with ELM, OS-ELM and WOS-ELM in forecast accuracy and stability. By comparing with these three algorithms, test the MM-WOS-ELM prediction performance of the algorithm in this paper.

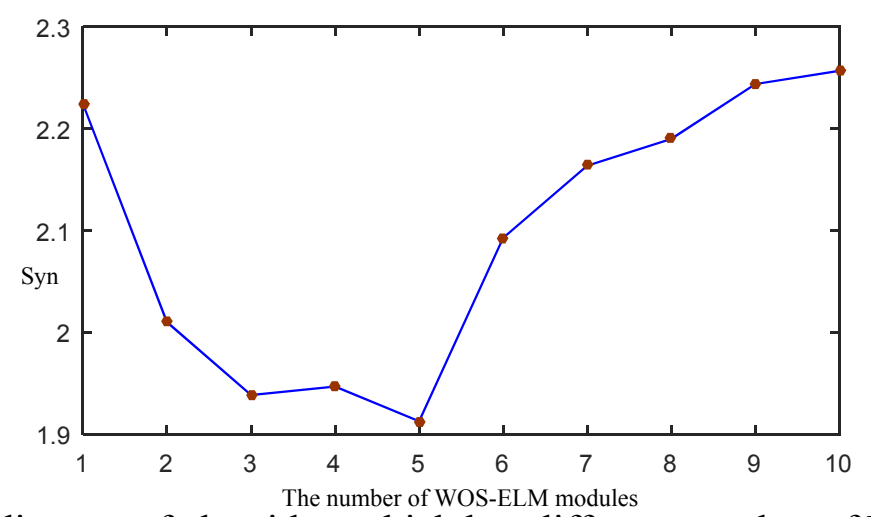

Fig.2. Integrated indicators of algorithm which has different number of WOS-ELM modules

For confirming the number of WOS-ELM modules through test data, this experiment took calculating comprehensive index as the objective function. In order to ensure the objectivity of the experiment, the number of modules was tested from 1 to 10 one hundred times, and used the average as the final result. The relationship between the number of modules and integrated indicators is shown in Figure 2. As it is shown in Figure 2, when the module number is 5, Syn is minimum. Therefore, the following experiments were carried out based on the number of modules is 5 .

Observe the algorithm prediction accuracy through RMSE. In order to ensure the accuracy and objectivity of predictions, experimented 50 times and the average value as a result of each algorithm. The RMSE of remaining load forecasting by using 4 algorithms is shown in Table 1. From Table 1, it can be seen that the RMSE of MM-WOS-ELM is minimum. It means that the performance of MM-WOS-ELM is better than ELM, OS-ELM, WOS-ELM. 
Test data was used as predicted data which was from 18:45 on April 3, 2012 to the next day 2:00. Figure 3 are the charts which MM-WOS-ELM compared with ELM, OS-ELM and WOS-ELM respectively.

Tab.1. Perdiction RMSE based on MM-WOS-ELM, OS-ELM, OS-ELM and WOS-ELM in test samples

\begin{tabular}{cl}
\hline Prediction Algorithm & RMSE \\
\hline MM-WOS-ELM & 2.2956 \\
ELM & 2.7976 \\
OS-ELM & 2.4817 \\
WOS-ELM & 2.3620 \\
\hline
\end{tabular}

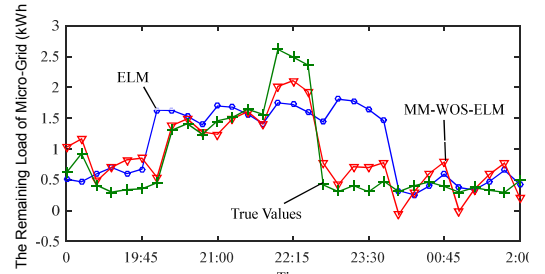

(a)ELM

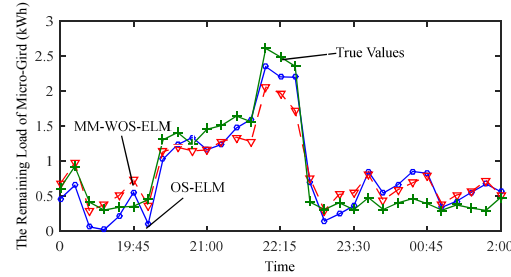

(b)OS-ELM

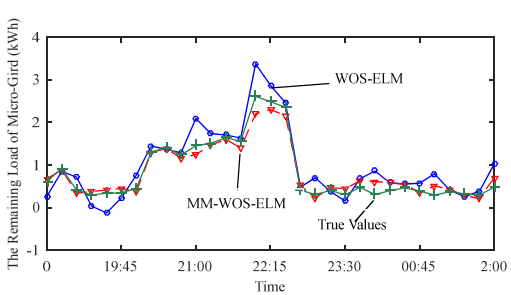

(c)WOS-ELM

Fig.3. Comparisons of the actual uncontrollable remaining load profile and the predicted ones by MM-WOS-ELM and others from 18:45 on April 3th, 2012 to 2:00 next day

Predict stability was calculated by using the Standard Deviation of RMSE. And stability of MM-WOS-ELM was compared with that of ELM, OS-ELM and WOS-ELM. This experimented 100 times to obtain the value of each of the RMSE and the average of these values as Table 2. From Table 2, the Standard Deviation of MM-WOS-ELM is minimum and that of ELM is maximum.

Tab.2. Comparisons of the Stability of RMSE based on MM-WOS-ELM, OS-ELM, OS-ELM and WOS-ELM in test samples

\begin{tabular}{cc}
\hline Prediction Algorithm & DS of RMSE \\
\hline MM-WOS-ELM & 0.0435 \\
ELM & 0.1324 \\
OS-ELM & 0.0950 \\
WOS-ELM & 0.1049 \\
\hline
\end{tabular}

\section{Conclusion}

With the development of distributed energy, as well as the popularity of the smart grid, micro-grid has also gradually become the research target of people. Micro-grid which is an important part in the field of smart grid, has broad application prospects in the commercial and industrial area, city area and in remote areas. However, the investment and operational cost of micro-grid are higher. So we need to balance the power load and the generation of micro-power supply, and develop appropriate power plan to make the lowest cost. This article in view of the remaining load forecasting, puts forward the prediction algorithm of MM-WOS-ELM, which not only can accurately predict the relationship between the power load and the uncontrolled generation of micro-power supply, but also improve the stability of prediction.

\section{Acknowledgement}

In this paper, the research was sponsored by the National Natural Science Foundation of China (Project No. 61300040) and Scientific Research Project of Hebei Province (Project No. Z2012077).

\section{References}

[1] Beshr, E., Comparative Study of Adding PV/Wind Energy Systems to Autonomus Micro Grid[C], 2013 3rd International Conference on Electric Power and Energy Conversion Systems, pp.1-6, 2-4 Oct. 2013. 
[2] Baoquan Liu and so on, System Operation and Energy Management of a Renewable Energy-Based DC Micro-Grid for High Penetration Depth Application[J], IEEE Transactions on Smart Grid, vol.6, no.3, pp.1147-1155, May 2015.

[3] Zhu Bo, Strategies for Micro-Grid Economic Operation based on Power Generation and Short-Term Load Forecasting [D]. Chongqing University, 2012.

[4] Jiang Qiyun, System Control Technology Research of Micro-Grid [D]. Zhejiang University, 2014.

[5] Gang Yan; Gao-hui Tang; Ji-ming Xiong, Electric Load Forecasting based on Improved LS-SVM Algorithm[C], 2012 10th World Congress on Intelligent Control and Automation (WCICA), pp.3064-3067, 6-8 July 2012.

[6] Kouhi S, Keynia F, Ravadanegh S N, A New Short-Term Load Forecast Method based on Neuro-Evolutionary Algorithm and Chaotic Feature Selection [J]. Electrical Power and Energy Systems, 2014, 62:862-867.

[7] Huang G B, Zhu Q Y, Siew C K, Extreme Learning Machine: a New Learning Scheme of Feed-Forward Neural Networks[C]//2004 IEEE International Joint Conference on Neural Networks. Budapest, Hungary: IEEE, 2004: 985-990.

[8] Huang Ting and so on, Short-Term Wind Power Prediction base on the Wavelet-Extreme Learning Machine [J]. Control Engineering, 2012,S1: 232-236.

[9] Yang Lei, Prediction Research of the Output Power of Solar Photovoltaic Power Plant [D]. Lanzhou Jiaotong University, 2014.

[10]Wang Baoyi and so on, The Distributed Power Load Forecasting Algorithm base on Cloud Computing and Ultimate Learning Machine [J]. Power System Technology, 2014, 38(2): 526-531.

[11]Nan-Ying Liang; Guang-Bin Huang; Saratchandran, P.; Sundararajan, N., A Fast and Accurate Online Sequential Learning Algorithm for Feed-forward Networks[J], IEEE Transactions on Neural Networks, vol.17, no.6, pp.1411-1423, Nov. 2006.

[12] Sun Yigang; Liu Jingya; Zhao Zhen, Weighted Sequential Limit Online Learning Machine Algorithm and its Application [J]. Computer Engineering and Design, 2014, 10:3594-3597+3666.

[13]Chen Minyou and so on, Micro-Grid Remaining Ultra-Short Term Load Forecasting based on Hybrid Intelligent Technology [J]. Electric Power Automation Equipment, 2012, 32(5): 13-18.

[14]Ma Yiwei; Yang Ping and so on, The Optimum Design of Islands Hybrid Renewable Energy Systems [J]. South China University of Technology (Natural Science), 2012, 11:113-120.

[15]Mohamed F A, Micro-Grid Modeling and Online Management in Department of Automation and Systems Technology [D]. Espoo, Finland: Department of Automation and Systems Technology, Helsinki University of Technology, 2008: 169. 